\title{
Developmental Origins of Cardiovascular Disease
}

\author{
Edwina H. Yeung • Candace Robledo • \\ Nansi Boghossian • Cuilin Zhang • Pauline Mendola
}

Published online: 18 January 2014

(C) Springer International Publishing AG (outside the USA) 2014

\begin{abstract}
Although cardiovascular disease has traditionally been viewed as a condition of aging individuals, increasing focus has turned to its developmental origins. Since birth weight has been related to cardiovascular disease risk, research into factors such as gravid conditions that affect fetal growth have grown. Associations between maternal diabetes and childhood obesity from sibling studies suggest a causal role, but prospective studies of gestational diabetes remain mixed. Preeclampsia and increased offspring blood pressure have been consistently observed, but evidence for other cardiovascular outcomes is lacking. While maternal obesity is associated with childhood obesity, causality remains unclear and paternal obesity should be investigated as an independent risk factor. Environmental chemical exposures in utero, particularly obesogens, are now emerging as another concern, as is conception via infertility treatment. Few studies have investigated the subclinical measures of endothelial function or atherosclerosis, and more research in these areas may help to reveal the underlying pathogenesis.
\end{abstract}

\footnotetext{
E. H. Yeung $(\bowtie) \cdot$ C. Robledo $\cdot$ N. Boghossian $\cdot$ C. Zhang $\cdot$

P. Mendola

Epidemiology Branch, Division of Intramural Population Health Research, Eunice Kennedy Shriver National Institute of Child Health and Human Development, 6100 Executive Blvd, 7B03,

Bethesda, MD 20892, USA

e-mail: yeungedw@mail.nih.gov

C. Robledo

e-mail: robledoc@mail.nih.gov

N. Boghossian

e-mail: nansi.boghossian@nih.gov

C. Zhang

e-mail: zhangcu@mail.nih.gov

P. Mendola

e-mail: pauline.mendola@nih.gov
}

Keywords Developmental origins - Cardiovascular disease Birth weight · Preterm birth · Gestational diabetes .

Preeclampsia $\cdot$ Maternal obesity $\cdot$ Environmental chemicals

\section{Introduction}

Cardiovascular disease (CVD) and stroke remain the leading cause of mortality in the United States, accounting for 1 in 3 deaths in 2009 [1]. With the strategic goal of decreasing CVD and stroke mortality rates by $20 \%$ by 2020 , the American Heart Association has highlighted the need for "primordial" prevention at the population level, aiming to shift the distribution of CVD risk factors of all Americans rather than relying on individual treatments or secondary prevention [2]. Although smoking, poor diet, and low physical activity are well-known modifiable factors related to CVD risk, emerging evidence has pointed to even earlier origins where populationlevel preventive strategies could be targeted. In particular, prenatal factors have been associated with later childhood or adulthood obesity, diabetes, and hypertension, all of which contribute to CVD risk. Therefore, this review serves to summarize the evidence to date for the developmental origins of cardiovascular disease in relation to birth outcomes, pregnancy complications, and other under-researched areas of earlylife influence.

\section{Birth Outcomes}

The association between blood pressure in adulthood and birth weight has been noted since the 1980s [3, 4], and the developmental origins of health and disease hypothesis (DOHaD) has become widely accepted [5]. It is hypothesized that fetal adaptations made in response to undernourishment lead to permanent changes in the body's structure and function that increase long-term risks of chronic diseases such as CVD [6]. 
Since then, many studies around the world have replicated these associations. In meta-analyses, each $1 \mathrm{~kg}$ increase in birth weight decreased cardiovascular mortality by $9-15 \%$ [7] and decreased systolic blood pressure by $2-4 \mathrm{mmHg}[8,9]$. Recently, the Bogalusa Heart Study with detailed data on measured blood pressure from adults aged 19-50 years further added to the evidence that low birth weight not only increased systolic and diastolic blood pressure, but also influenced blood pressure variability, suggesting altered regulation [10].

Nevertheless, findings have been conflicting regarding low birth weight and subclinical markers of atherosclerosis, as previously reviewed [11] and among more recent studies [12-14]. Some studies have found positive rather than negative or U-shaped associations with birth weight, suggesting a stronger influence of later-life overnutrition or hyperglycemia on later atherosclerotic development [12]. Others have found no association [11], but the modeling of postnatal influences is complex and studies vary in methods used to account for current lifestyle risk factors and, in particular, current size. One concern is that current size lies in the pathway from birth weight to CVD risk, such that controlling for the intermediate of current size generates bias in the estimates of the associations. Using more current epidemiologic methods [15], a recent analysis demonstrated that the negative association between birth weight and blood pressure after adjustment for current weight is unlikely to be caused by controlling for the possible intermediate of current weight [16]. Rather, it is likely a direct effect of birth weight on systolic blood pressure that is not mediated by current weight [16]. Such analyses are reassuring that current size can be accounted for in statistical models.

Another possible explanation for the conflicting findings may be due to differences in treatment of gestational age in analyses, as low birth weight may arise due to either preterm birth or growth restriction. In a recent meta-analysis of studies measuring metabolic syndrome factors in adults, systolic blood pressure was $4.18 \mathrm{mmHg}$ higher among the preterm ( $<37$ weeks gestational age) compared to the term group [17]. Increases were also seen for diastolic blood pressure $(+2.57 \mathrm{mmHg})$ and low-density lipoprotein $(+0.14 \mathrm{mmol} / \mathrm{l})$ [17]. However, studies were heterogeneous in methods of selecting on preterm birth, and there is a mix of those who were appropriate for gestational age and those small for gestational age that does not clearly tease apart the effect of gestational age from fetal growth. In the subgroup analyses, the authors found that the blood pressure association was stronger among studies that recruited based on gestational age rather than birth weight [17]. The Cardiovascular Risk in Young Finns Study was one of the largest cohorts to try to tease these aspects apart. It compared measures of carotid intima-media thickness (IMT) and brachial flow-mediated dilation (FMD) at ages 21 and 27 years among a group of 207 participants who were born small for gestational age (i.e.,
$<10$ th percentile in birth weight by gestational age), a group of 253 participants who were born preterm $(<37$ weeks gestation), and 835 controls who were born at term and in the normal range of weight for gestational age (i.e., 50-90th percentile birth weight) [14]. Brachial FMD was lower and carotid IMT was higher among those born small for gestational age, with similar results among the term and the preterm groups [14]. Both lower FMD and higher IMT are related to greater CVD risk, with FMD being related to endothelial dysfunction and IMT as an indicator of subclinical atherosclerosis [18]. Further study is necessary before a conclusion can be drawn regarding whether preterm birth has an effect that is independent from birth size.

Even fewer studies have data on fetal growth as measured by ultrasound and postnatal development [19]. However, as recently systematically reviewed, a handful of studies have consistently observed inverse associations between fetal growth measures (such as abdominal circumference) and later systolic blood pressure [19]. Future studies tracking the longterm health of children with such data from pregnancy may be helpful for understanding the association between fetal growth trajectory and later health, as ultrasound measures are routine in obstetric practice. Interest remains in whether or not the increased ability of the fetus to accumulate mass in utero as measured by growth velocity could serve as an indicator for later susceptibility to a high-caloric, low-physical-activity environment.

Placental inadequacies resulting in fetal malnutrition have also been implicated in adult cardiovascular disease in offspring [20]. Among 2,571 men born in Sheffield, UK, mortality from coronary heart disease showed a U-shaped association with the placental-to-birth weight ratio, the highest mortality being at either end of the distribution [21]. A high placental-to-birth weight ratio also predicted cardiovascular disease mortality among 31,000 men and women in Norway; the sex and cohort-adjusted hazard ratio for the highest versus the lowest third of the placenta-to-birth weight ratio was 1.38 (95\% CI: 1.07 to 1.77) [22]. The Helsinki birth cohorts have been critical in uncovering several of the relationships between placental size and cardiovascular diseases in adulthood. Among women who were born full-term and compared to those who were longest at birth $(>50 \mathrm{~cm})$ with had the lowest placental weight $(\leq 500 \mathrm{~g})$, women who were short at birth $(\leq 48 \mathrm{~cm})$ but had heavy placentas $(>700 \mathrm{~g})$ had the highest coronary heart disease hazard ratio of 5.2 [23]. These comparisons suggest that a heavier placenta in relation to birth length could be an indicator of fetal malnutrition being inadequately compensated for by placental growth. The association differed by gender. Among men, a low placental weight reflected thinness by a low ponderal index and increased mortality from coronary heart diseases later in life [24]. Altered patterns of prenatal growth may have subsequent sexdependent consequences on coronary heart disease risks. 
Other characteristics of placental morphology may also serve as indicators of fetal malnutrition [20]. In a study of 2,003 subjects, of whom 644 were being treated for hypertension, hypertension was associated with decreased placental weight and surface area at birth [25]. Another investigation of the Helsinki Birth Cohort identified that a thin placenta was associated with increased risk of sudden cardiac death, suggesting impaired autonomic nervous development [26]. Surface area was estimated by the measurement of its two diameters, assuming that the placenta was of elliptical shape. Thickness was then taken to be placental weight divided by estimated surface area. In following up with these observations regarding placental surface area, investigators counted the number of placental cotyledons from photographs of placentas from 910 subjects in a UK birth cohort with measured blood pressure at age 9 years [27]. Contrary to their hypothesis, however, an increased number of cotyledons correlated with increased blood pressure [27]. Such findings suggest there is much to learn about placental morphology and function with fundamental questions remaining related to the mechanisms underlying the placental origins of later heart disease in offspring [28]. Difficulties with measuring accurate placental weight and other morphological features in large epidemiologic studies may remain a research hurdle that should be addressed with particular attention to methodology in study design.

\section{Pregnancy Complications}

Not only are the effects of gestational age apart from fetal growth and placental morphology important considerations, upstream causes of each may shed more light on the associations found between birth outcomes and CVD. Moreover, these upstream factors provide opportunities for potential intervention. As such, studies have also investigated the associations between CVD in the offspring for whom birth was complicated by various maternal gravid conditions. Most frequently, studies have focused on gestational diabetes and preeclampsia. Such pregnancy complications could suggest an unfavorable intrauterine environment.

\section{Gestational Diabetes Mellitus}

Gestational diabetes mellitus (GDM), defined as glucose intolerance with onset or first detection in pregnancy, occurs in approximately 4-7\% of all pregnancies in the USA [29]. Epidemiologic studies have shown conflicting evidence for the association between GDM and childhood obesity. Variations in diagnosis criteria, severity, and treatment of GDM, and in age and measurement of childhood adiposity may at least partly account for inconsistencies of the findings. Most of the evidence of the association between GDM and childhood obesity is from prospective cohort studies or retrospective cohorts with medical record information, which are advantageous in not relying on maternal recall of GDM status years after delivery. However, inconsistencies may be partly due to the age of children studied and high attrition rates decreasing statistical power to detect differences [30, 31]. Studies among infants and younger children who may not have experienced their adiposity rebound do not show any difference in BMI with respect to exposure to maternal hyperglycemia [32-34]. One trial also showed no significant difference in childhood overweight at 4-5 years of age among mothers who were actively treated for mild GDM and those who were not treated [35]. The measure of adiposity may also affect results, with one study finding an association using skinfolds [36], but not BMI, while another found a stronger association with waist circumference than BMI [37]. Studies have found that adjustment for pre-pregnancy body mass index (BMI) attenuates [38], and in some cases eliminates, the association altogether $[39,40]$.

Universal screening for GDM was not recommended until recently, making it difficult to ensure correct classification of exposure status among children born in earlier periods. Moreover, hyperglycemia below the threshold for GDM diagnosis may still prove detrimental for fetal outcomes [41]. Studies have therefore also begun to investigate the level of hyperglycemia during pregnancy rather than comparing only GDM versus non-GDM [32, 42-44]. Of these studies, the Center for the Health Assessment of Mothers and Children of Salinas (CHAMACOS) study had the longest follow-up, finding that waist circumference at age seven was significantly associated with continuous glucose measures during pregnancy, even after adjusting for maternal pre-pregnancy BMI [42].

Studies may be limited by confounding from lifestyle patterns such as maternal diet and physical activity that are shared with the offspring in childhood. Few sibling studies have been conducted to try to address the issue of genetics and shared lifestyle factors $[45,46]$. However, neither study was restricted to gestational diabetes. The first was a study among Pima Indian mothers in the late 1990s [45]. Another was recently conducted among 280,866 singleton-born Swedish men [46]. Using record linkages with military conscription data at age 18 years, the men and their brothers were compared for differences in BMI by maternal diabetes exposure with no distinction made between gestational and pregestational diabetes. A mean BMI increase of $1.23(95 \%$ CI: 0.11 to 2.36$) \mathrm{kg} / \mathrm{m}^{2}$ was found for the men exposed to maternal diabetes compared to their non-exposed brothers, after adjusting for various risk factors including earlypregnancy BMI. The difference in BMI was attenuated between non-siblings discordant for exposure $\left(0.41 \mathrm{~kg} / \mathrm{m}^{2} ; 95 \%\right.$ CI: 0.15 to 0.67$)$, but remained significant, suggesting that the association is neither explained by genetics nor postnatal environmental exposures, but rather is strongly related to the intrauterine environment. However, associations for GDM or lower levels of hyperglycemia remain untested. 
With regard to other cardiovascular disease indicators and GDM, a meta-analysis found that GDM was associated with slight increase in systolic $(+1.39 \mathrm{mmHg})$, but not diastolic blood pressure in offspring measured in childhood or adolescence [47]. Findings regarding lipid metabolism have been divided, with some finding no association $[42,48]$ and others detecting a difference [30,37], although associations may be driven by concurrent findings of increased childhood BMI. One study from Hong Kong found no differences in arterial stiffness among 42 GDM-exposed adolescent offspring at the age of 15 years compared to 87 controls [49]. However, cord blood levels of c-peptide were positively associated with carotid-femoral pulse wave velocity (i.e., an indicator for arterial stiffness) and other vascular measures [49]. More studies are needed on the endothelial function of children exposed to GDM.

\section{Preeclampsia}

Preeclampsia is generally defined as new onset hypertension during pregnancy accompanied by proteinuria after 20 weeks of gestation, although no consensus exists for its diagnosis and its pathogenesis remains unclear [50]. It can affect $2-8 \%$ of pregnancies and is the leading cause of both maternal and perinatal mortality and morbidity [50]. Similar to GDM, maternal obesity is a known risk factor for the development of preeclampsia, making prepregnancy BMI a potentially strong confounder of any relationships with childhood CVD risk. As earlier delivery at 34-36 weeks occurs frequently for pregnant women with preeclampsia [51], associations with offspring CVD risk factors may be mediated by gestational age. Davis et al. systematically reviewed studies available up to August 2011 and determined in a meta-analysis of 12 studies that blood pressure is elevated among offspring (aged 4-30 years) born to women who had preeclampsia compared to controls $(+2.39 \mathrm{mmHg}$ SBP and $+1.35 \mathrm{mmHg}$ DBP) [52]. In a sensitivity analysis restricted to four studies with information on gestational age, findings remained similar for term infants of normal birth weight [52]. Offspring BMI also differed $\left(+0.62 \mathrm{~kg} / \mathrm{m}^{2}\right)$ by preeclampsia status and a few studies showed increased triglyceride and lower HDL cholesterol [52]. Since Davis' meta-analysis, findings from two longterm longitudinal studies with measures at age 16 years [53] and 17 years [54] further confirmed positive associations with blood pressure. Gestational hypertension without preeclampsia was also found to increase childhood blood pressure [53, 54].

With regard to endothelial dysfunction, a pilot study of 26 mother-child pairs with preeclampsia compared to 17 healthy pairs measured reactive hyperemia after occlusion of the brachial artery among children at age 5-8 years of age [55] No differences were found by preeclampsia status, but instead a lower reactive hyperemia index was found among those born SGA (which included cases and controls). However, larger studies are needed to replicate these findings. Some research hurdles include difficulties in classifying preeclampsia severity, and time of diagnosis may play a role in how the fetus is affected.

\section{Maternal Adiposity}

Associations between maternal adiposity and childhood obesity have been systematically reviewed [56]. Although many studies have shown an association, some doubt has been cast on whether the association is purely due to genetic or shared environmental effects [57-59]. One argument is that maternal obesity should have stronger associations than paternal obesity if "programming" occurs due to prenatal influences of increased maternal adiposity on the fetal endocrine system, or other structures. Observations that associations are similar in magnitude between paternal and maternal BMI and risk of childhood obesity then suggest that the associations are due to shared postnatal lifestyle $[58,59]$. What is not clear is whether paternal adiposity plays a distinct role in "programming" as well. Preliminary evidence in a mouse model suggests that paternal diet-induced obesity transmitted obesity through two generations of offspring with epigenetic changes detected [60].

Adding to the complexity of this association is that obesity may be associated with many different nutritional influences. Most epidemiologic studies have used gestational weight gain as a proxy for overconsumption during pregnancy. The amount of gestational weight gain through pregnancy is the sum of three primary components involving maternal tissue, placenta, and fetus [61]. A study of $\sim 140,000$ sibling pairs found that maternal fat gain (measured by the difference between postnatal weight and weight at first clinic assessment) among overweight and obese women was associated with a small increase in offspring BMI at age 18 years $(0.06 \mathrm{~kg} / \mathrm{m} 2 ; 95 \%$ : 0.01-0.12), with no additional contribution of weight gain among normal weight women [62]. Timing of the weight gain may also be important to decipher, with one study showing greater effects on offspring development found for weight gained prior to the third trimester [63]. Fetal weight makes a greater contribution to third trimester weight, while earlier weight gain is more likely to reflect maternal fat stores. In terms of specific dietary factors, evidence remains insufficient for dietary recommendations during pregnancy directed at limiting childhood obesity [64], although some suggestions of fatty acid consumption having an impact on later offspring adiposity have been observed $[65,66]$.

\section{Environmental Exposures}

Evidence implicating environmental exposures on risk of cardiovascular disease is accumulating. Most notable is tobacco smoke, considered to be the most preventable cause of 
cardiovascular disease [67]. A well-established link also exists between long- [68] and short-term [69] exposure to ambient air pollutants and CVD. The evidence for long-term exposures causing increased cardiovascular mortality risk is strongest for fine particles, elemental carbon, and nitric oxides [68], which commonly result from traffic and other combustion sources. Exposure to other environmental chemical exposures from common sources such as pesticides or plastics is also fairly ubiquitous [70], and many of these chemicals can directly or indirectly influence the risk of cardiovascular disease.

That the fetal cardiovascular system is affected by maternal smoking has long been recognized [71]. More recently, the National Toxicology Program reviewed the evidence regarding its positive association with childhood obesity, concluding that it is most likely causal [72]. Too few epidemiologic studies, however, have studied secondhand smoking exposure in relation to offspring cardiovascular disease risk factors. A study of over 74,000 pregnant Norwegian women between the ages of 14-44 years found that in utero tobacco smoke exposure (i.e., during their mothers' pregnancy) was associated with increased odds of obesity $(\mathrm{OR}=1.53,95 \% \mathrm{CI}: 1.45$ 1.61), hypertension ( $\mathrm{OR}=1.68,95 \% \mathrm{CI}: 1.19-2.39)$, and gestational diabetes mellitus $(\mathrm{OR}=1.32,95 \% \mathrm{CI}: 1.10-1.58)$ [73]. This study suggests that smoking exposure may have transgenerational effects through other pathways, even if smoking itself is discontinued in the next generation.

Numerous studies have linked the risk of preterm delivery and growth restriction to maternal exposure to air pollution and environmental chemicals (e.g., persistent organic pollutants such as polychlorinated bi-phenyls (PCBs), metals such as lead, non-persistent chemicals such as bisphenol-A) [74, 75]. As discussed earlier, these birth outcomes have long-term implications on cardiovascular disease risk and health. Whether there is an independent direct effect of early-life air pollutant exposure on subsequent CVD risk is unknown, but the developmental effects or "programming" of respiratory anatomy and function by early-life air pollutant exposure has been suggested, perhaps as a result of oxidative stress [76]. Cognitive effects have also been proposed as a result of the proinflammatory responses common in children exposed to air pollution, such as high levels of inflammatory cytokines and associated tissue remodeling and regulatory immune responses [77]. As such, evidence from the respiratory and neurodevelopmental literature suggests that adult cardiovascular disease may also share early-life air pollution exposure risk driven by the developmental effects of inflammation and oxidative stress. This understudied area merits further investigation.

Other exposures may act on offspring CVD risk indirectly, particularly by increasing obesity risk. Some environmental chemicals can act as endocrine active compounds that either mimic or interfere with the normal function of hormones. A fast-growing area of research is the identification of "obesogens" or environmental chemicals, such as phthalates [78] and bisphenol-A [79], that may promote obesity through mechanisms of lipid regulation and adiopogenesis. Prenatal exposure to PCBs has been associated with heavier children [74] and changes in offspring growth and development have been observed for both prenatal and lactational exposure [80]. Similar to the notion of fetal "programming" derived from maternal nutrition, these chemical obesogens may result in permanent changes not detectable until later in life. These changes can impact the function and response of hormones, which can ultimately promote obesity in the offspring [81]. Two systematic reviews evaluated the evidence for the association between environmental chemicals and increased adiposity from chemical exposure at various ages [82, 83]. Most studies found a significant positive association between adiposity and the organochlorine insecticide, dichlorodiphenyltrichloroethane and its metabolite. Research in this area is complicated because the effects of environmental chemicals on growth and development may not follow traditional monotonic dose-response curves, the response may differ between offspring and parents, or in utero effects may not be evident until adulthood.

To our knowledge, prenatal chemical exposures have not been studied in association with markers of atherosclerosis or other hard CVD outcomes in offspring. However, adulthood exposure to bisphenol-A was associated with the severity of coronary artery disease, as measured by angiography in one cross-sectional study of 591 adults from the UK [84]. Whether there are persistent effects from in utero exposure remains to be seen, and the impact of environmental chemical exposures on cardiovascular disease health outcomes and co-morbidities should not be overlooked.

\section{Infertility Treatment}

Infertility treatments, particularly assisted reproductive technologies (ART), are associated with a higher risk of preterm birth and low birth weight along with pregnancy complications [85]. As its worldwide use has been increasing [86-88], conception by ART may serve as an important risk factor for later cardiovascular risk. Three of five studies have found that children conceived by infertility treatment have higher blood pressure than those conceived without treatment [89]. Two studies found increased carotid IMT among children conceived by ART compared to controls (measured at 6 months [90] and at 11 years [91], respectively) along with other vascular differences. One of the studies compared children who did not differ by gestational age or birth weight [91], whereas the other study included 17 low-birth-weight children in the ART group compared to one child in the control group and adjusted for birth weight percentile along with gestational age and preeclampsia [90]. Epigenetic alterations to vasculature due to ART treatment, perhaps resulting from in vitro 
culture, sperm selection, or ovarian stimulation, have been hypothesized to be an underlying mechanism behind the observed vascular differences [91, 92]. Methylation patterns of placental and cord blood samples significantly differ among those conceived by ART and those not, particularly for genes in the adiposity and metabolic pathways [93]. These methylation differences require confirmation, and larger studies with longitudinal follow-up are necessary to better understand whether these observations persist through the life course and are not confounded by shared lifestyle factors or a common cause associated with underlying infertility.

\section{Conclusions}

More remains to be done to identify the "primordial" risk factors and inter-generational effects that can be the next frontier of modifiable CVD risk. Women who are planning pregnancy may be strongly motivated to make lifestyle changes that will benefit the long-term health of their offspring. However, the impact of the paternal contribution should not be dismissed. Whether observed associations are truly causal will remain a difficult task for epidemiologists to decipher, but new techniques for teasing apart the direct and indirect effects that impact the individual contributions of birth outcomes, their preceding gravid conditions, and the broader influences of nutrition and environmental exposures appear promising. In spite of the complexity of the causal relationships between pregnancy complications, parental obesity, and CVD outcomes in the children, those complications may serve as a vehicle to help identify children who are at high risk.

\section{Compliance with Ethics Guidelines}

Disclosure All work was supported by the Intramural Research Program of the Eunice Kennedy Shriver National Institute of Child Health \& Human Development, National Institutes of Health.

Conflict of Interest EH Yeung declares no conflicts of interest.

$\mathrm{C}$ Robledo declares no conflicts of interest.

$\mathrm{N}$ Boghossian declares no conflicts of interest.

C Zhang declares no conflicts of interest.

P Mendola declares no conflicts of interest.

Human and Animal Rights and Informed Consent This article does not contain any studies with human or animal subjects performed by any of the authors.

\section{References}

1. Go AS, Mozaffarian D, Roger VL, et al. Executive summary: heart disease and stroke statistics-2013 update: a report from the American Heart Association. Circulation. 2013;127(1):143-52.

2. Lloyd-Jones DM, Hong Y, Labarthe D, et al. Defining and setting national goals for cardiovascular health promotion and disease reduction: the American Heart Association's strategic Impact Goal through 2020 and beyond. Circulation. 2010;121(4): 586-613.

3. Barker DJ, Osmond C. Low birth weight and hypertension. BMJ. 1988;297(6641):134-5.

4. Wadsworth ME, Cripps HA, Midwinter RE, et al. Blood pressure in a national birth cohort at the age of 36 related to social and familial factors, smoking, and body mass. Br Med J (Clin Res Ed). 1985;291(6508):1534-8.

5. Paul AM. Fetal origins: how the first nine months shape your life. Time. 2010.

6. Barker DJ. Fetal origins of coronary heart disease. Br Heart J. 1993;69(3):195-6.

7. Risnes KR, Vatten LJ, Baker JL, et al. Birthweight and mortality in adulthood: a systematic review and meta-analysis. Int J Epidemiol. 2011;40(3):647-61.

8. Law CM, Shiell AW. Is blood pressure inversely related to birth weight? The strength of evidence from a systematic review of the literature. J Hypertens. 1996;14(8):935-41.

9. Mu M, Wang SF, Sheng J, et al. Birth weight and subsequent blood pressure: a meta-analysis. Arch Cardiovasc Dis. 2012;105(2):99 113.

10. Chen W, Srinivasan SR, Yao L, et al. Low birth weight is associated with higher blood pressure variability from childhood to young adulthood: the Bogalusa Heart Study. Am J Epidemiol. 2012;176 Suppl 7:S99-S105.

11. Norman M. Low birth weight and the developing vascular tree: a systematic review. Acta Paediatr. 2008;97(9):1165-72.

12. Dratva J, Breton CV, Hodis HN, et al. Birth weight and carotid artery intima-media thickness. J Pediatr. 2013;162(5):906-11.

13. Hovi P, Turanlahti M, Strang-Karlsson S, et al. Intima-media thickness and flow-mediated dilatation in the Helsinki study of very low birth weight adults. Pediatrics. 2011;127(2):e304-11.

14. Skilton MR, Viikari JS, Juonala M, et al. Fetal growth and preterm birth influence cardiovascular risk factors and arterial health in young adults: the Cardiovascular Risk in Young Finns Study. Arterioscler Thromb Vasc Biol. 2011;31(12):2975-81.

15. VanderWeele TJ. Bias formulas for sensitivity analysis for direct and indirect effects. Epidemiology. 2010;21(4):540-51.

16. Chiolero A, Paradis G, Kaufman JS. Assessing the possible direct effect of birth weight on childhood blood pressure: a sensitivity analysis. Am J Epidemiol. 2013;179:4-11.

17. Parkinson JR, Hyde MJ, Gale C, et al. Preterm birth and the metabolic syndrome in adult life: a systematic review and meta-analysis. Pediatrics. 2013;131(4):e1240-63.

18. Snell-Bergeon JK, Nadeau K. Cardiovascular disease risk in young people with type 1 diabetes. J Cardiovasc Transl Res. 2012;5(4):44662.

19. Norris T, Cameron N. Investigating the relationship between prenatal growth and postnatal outcomes: a systematic review of the literature. J Dev Origins Health Dis. 2013;4(6):434-41.

20. Barker DJ, Thornburg KL. Placental programming of chronic diseases, cancer and lifespan: a review. Placenta. 2013;34(10):841-5.

21. Martyn CN, Barker DJ, Osmond C. Mothers' pelvic size, fetal growth, and death from stroke and coronary heart disease in men in the UK. Lancet. 1996;348(9037):1264-8.

22. Risnes KR, Romundstad PR, Nilsen TI, et al. Placental weight relative to birth weight and long-term cardiovascular mortality: findings from a cohort of 31,307 men and women. Am J Epidemiol. 2009; 170(5):622-31.

23. Forsen T, Eriksson JG, Tuomilehto J, et al. Growth in utero and during childhood among women who develop coronary heart disease: longitudinal study. BMJ. 1999;319(7222):1403-7.

24. Forsen T, Eriksson JG, Tuomilehto J, et al. Mother's weight in pregnancy and coronary heart disease in a cohort of Finnish men: follow up study. BMJ. 1997;315(7112):837-40. 
25. Barker DJ, Thornburg KL, Osmond C, et al. The surface area of the placenta and hypertension in the offspring in later life. Int J Dev Biol. 2010;54(2-3):525-30.

26. Barker DJ, Larsen G, Osmond C, et al. The placental origins of sudden cardiac death. Int J Epidemiol. 2012;41(5):1394-9.

27. Barker D, Osmond C, Grant S, et al. Maternal cotyledons at birth predict blood pressure in childhood. Placenta. 2013;34(8):672-5.

28. Thornburg KL, O'Tierney PF, Louey S. Review: the placenta is a programming agent for cardiovascular disease. Placenta. 2010;31(Suppl):S54-9.

29. Gestational diabetes mellitus. Diabetes Care. 2004;27 Suppl 1:S88S90.

30. Tam WH, Ma RC, Yang X, et al. Glucose intolerance and cardiometabolic risk in children exposed to maternal gestational diabetes mellitus in utero. Pediatrics. 2008;122(6):1229-34.

31. Catalano PM, Farrell K, Thomas A, et al. Perinatal risk factors for childhood obesity and metabolic dysregulation. Am J Clin Nutr. 2009;90(5):1303-13.

32. Pettitt DJ, McKenna S, McLaughlin C, et al. Maternal glucose at 28 weeks of gestation is not associated with obesity in 2-year-old offspring: the Belfast Hyperglycemia and Adverse Pregnancy Outcome (HAPO) family study. Diabetes Care. 2010;33(6):1219-23.

33. Pham MT, Brubaker K, Pruett K, et al. Risk of childhood obesity in the toddler offspring of mothers with gestational diabetes. Obstet Gynecol. 2013;121(5):976-82.

34. Chatzi L, Papadopoulou E, Vafiadi M, et al. Maternal glucose at 28 weeks of gestation is not associated with obesity in 2-year-old offspring: the Belfast Hyperglycemia and Adverse Pregnancy Outcome (HAPO) family study: comment on Pettit et al. Diabetes Care. 2011;34(1):e10.

35. Gillman MW, Oakey H, Baghurst PA, et al. Effect of treatment of gestational diabetes on obesity in the next generation. Diabetes Care. 2010;33:964-8.

36. Wright CS, Rifas-Shiman SL, Rich-Edwards JW, et al. Intrauterine exposure to gestational diabetes, child adiposity, and blood pressure. Am J Hypertens. 2009;22(2):215-20.

37. Egeland GM, Meltzer SJ. Following in mother's footsteps? Motherdaughter risks for insulin resistance and cardiovascular disease 15 years after gestational diabetes. Diabet Med. 2010;27(3):257-65.

38. Lawlor DA, Fraser A, Lindsay RS, et al. Association of existing diabetes, gestational diabetes and glycosuria in pregnancy with macrosomia and offspring body mass index, waist and fat mass in later childhood: findings from a prospective pregnancy cohort. Diabetologia. 2010;53(1):89-97.

39. Gillman MW, Rifas-Shiman S, Berkey CS, et al. Maternal gestational diabetes, birth weight, and adolescent obesity. Pediatrics. 2003;111(3):e221-6.

40. Pirkola J, Pouta A, Bloigu A, et al. Risks of overweight and abdominal obesity at age 16 years associated with prenatal exposures to maternal prepregnancy overweight and gestational diabetes mellitus. Diabetes Care. 2010;33(5):1115-21.

41. Metzger BE, Lowe LP, Dyer AR, et al. Hyperglycemia and adverse pregnancy outcomes. N Engl J Med. 2008;358(19):1991-2002.

42. Ehrlich SF, Rosas LG, Ferrara A, et al. Pregnancy glycemia in Mexican-American women without diabetes or gestational diabetes and programming for childhood obesity. Am J Epidemiol. 2013;177(8):768-75.

43. Chandler-Laney PC, Bush NC, Rouse DJ, et al. Maternal glucose concentration during pregnancy predicts fat and lean mass of prepubertal offspring. Diabetes Care. 2011;34:741-5.

44. Knight B, Shields BM, Hill A, et al. The impact of maternal glycemia and obesity on early postnatal growth in a nondiabetic Caucasian population. Diabetes Care. 2007;30(4):777-83.

45. Dabelea D, Hanson RL, Lindsay RS, et al. Intrauterine exposure to diabetes conveys risks for type 2 diabetes and obesity: a study of discordant sibships. Diabetes. 2000;49(12):2208-11.
46. Lawlor DA, Lichtenstein P, Langstrom N. Association of maternal diabetes mellitus in pregnancy with offspring adiposity into early adulthood: sibling study in a prospective cohort of 280,866 men from 248,293 families. Circulation. 2011;123(3):258-65.

47. Aceti A, Santhakumaran S, Logan KM, et al. The diabetic pregnancy and offspring blood pressure in childhood: a systematic review and meta-analysis. Diabetologia. 2012;55(11):3114-27.

48. Vaarasmaki M, Pouta A, Elliot P, et al. Adolescent manifestations of metabolic syndrome among children born to women with gestational diabetes in a general-population birth cohort. Am J Epidemiol. 2009;169(10):1209-15.

49. Tam WH, Ma RC, Yip GW, et al. The association between in utero hyperinsulinemia and adolescent arterial stiffness. Diabetes Res Clin Pract. 2012;95(1):169-75.

50. Steegers EA, von Dadelszen P, Duvekot JJ, et al. Pre-eclampsia. Lancet. 2010;376(9741):631-44.

51. Sibai BM. Preeclampsia as a cause of preterm and late preterm (nearterm) births. Semin Perinatol. 2006;30(1):16-9.

52. Davis EF, Lazdam M, Lewandowski AJ, et al. Cardiovascular risk factors in children and young adults born to preeclamptic pregnancies: a systematic review. Pediatrics. 2012;129(6):e1552-61.

53. Miettola S, Hartikainen AL, Vaarasmaki M, et al. Offspring's blood pressure and metabolic phenotype after exposure to gestational hypertension in utero. Eur J Epidemiol. 2013;28(1):87-98.

54. Fraser A, Nelson SM, Donald-Wallis C, et al. Hypertensive disorders of pregnancy and cardiometabolic health in adolescent offspring. Hypertension. 2013;62(3):614-20.

55. Kvehaugen AS, Dechend R, Ramstad HB, et al. Endothelial function and circulating biomarkers are disturbed in women and children after preeclampsia. Hypertension. 2011;58(1):63-9.

56. Brisbois TD, Farmer AP, McCargar LJ. Early markers of adult obesity: a review. Obes Rev. 2012;13(4):347-67.

57. Lawlor DA, Relton C, Sattar N, et al. Maternal adiposity-a determinant of perinatal and offspring outcomes? Nat Rev Endocrinol. 2012;8(11):679-88.

58. Patro B, Liber A, Zalewski B, et al. Maternal and paternal body mass index and offspring obesity: a systematic review. Ann Nutr Metab. 2013;63(1-2):32-41.

59. Fleten C, Nystad W, Stigum H, et al. Parent-offspring body mass index associations in the Norwegian Mother and Child Cohort Study: a family-based approach to studying the role of the intrauterine environment in childhood adiposity. Am J Epidemiol. 2012;176(2): 83-92.

60. Fullston T, Ohlsson Teague EM, Palmer NO, et al. Paternal obesity initiates metabolic disturbances in two generations of mice with incomplete penetrance to the F2 generation and alters the transcriptional profile of testis and sperm microRNA content. FASEB J. 2013;27(10):4226-43.

61. IOM (Institute of Medicine) and NRC (National Research Council). Composition and components of gestational weight gain: physiology and metabolism. Weight gain during pregnancy: reexamining the guidelines. Washington, DC: National Academies Press; 2009.

62. Lawlor DA, Lichtenstein P, Fraser A, et al. Does maternal weight gain in pregnancy have long-term effects on offspring adiposity? A sibling study in a prospective cohort of 146,894 men from 136,050 families. Am J Clin Nutr. 2011;94(1):142-8.

63. Fraser A, Tilling K, Donald-Wallis C, et al. Association of maternal weight gain in pregnancy with offspring obesity and metabolic and vascular traits in childhood. Circulation. 2010;121(23):2557-64.

64. Symonds ME, Mendez MA, Meltzer HM, et al. Early life nutritional programming of obesity: mother-child cohort studies. Ann Nutr Metab. 2013;62(2):137-45.

65. Donahue SM, Rifas-Shiman SL, Gold DR, et al. Prenatal fatty acid status and child adiposity at age $3 \mathrm{y}$ : results from a US pregnancy cohort. Am J Clin Nutr. 2011;93(4):780-8. 
66. Mace K, Shahkhalili Y, Aprikian O, et al. Dietary fat and fat types as early determinants of childhood obesity: a reappraisal. Int J Obes (Lond). 2006;30 Suppl 4:S50-7.

67. Gastaldelli A, Folli F, Maffei S. Impact of tobacco smoking on lipid metabolism, body weight and cardiometabolic risk. Curr Pharm Des. 2010;16(23):2526-30.

68. Hoek G, Krishnan RM, Beelen R, et al. Long-term air pollution exposure and cardio- respiratory mortality: a review. Environ Health. 2013;12(1):43.

69. Shah AS, Langrish JP, Nair H, et al. Global association of air pollution and heart failure: a systematic review and meta-analysis. Lancet. 2013;382(9897):1039-48.

70. Centers for Disease Control and Prevention. Fourth Report on Human Exposure to Environmental Chemicals. 2009. Atlanta, GA, U.S. Department of Health and Human Services, Centers for Disease Control and Prevention. Ref Type: Report.

71. Asmussen I. Fetal cardiovascular system as influenced by maternal smoking. Clin Cardiol. 1979;2(4):246-56.

72. Behl M, Rao D, Aagaard K, et al. Evaluation of the association between maternal smoking, childhood obesity, and metabolic disorders: a national toxicology program workshop review. Environ Health Perspect. 2013;121(2):170-80.

73. Cupul-Uicab LA, Skjaerven R, Haug K, et al. In utero exposure to maternal tobacco smoke and subsequent obesity, hypertension, and gestational diabetes among women in the MoBa cohort. Environ Health Perspect. 2012;120(3):355-60.

74. Ferguson KK, O'Neill MS, Meeker JD. Environmental contaminant exposures and preterm birth: a comprehensive review. J Toxicol Environ Health B Crit Rev. 2013;16(2):69-113.

75. Mattison DR. Environmental exposures and development. Curr Opin Pediatr. 2010;22(2):208-18.

76. Wright RJ, Brunst KJ. Programming of respiratory health in childhood: influence of outdoor air pollution. Curr Opin Pediatr. 2013;25(2):232-9.

77. Calderon-Garciduenas L, Mora-Tiscareno A, Styner M, et al. White matter hyperintensities, systemic inflammation, brain growth, and cognitive functions in children exposed to air pollution. J Alzheimers Dis. 2012;31(1):183-91.

78. Janesick A, Blumberg B. Minireview: PPARgamma as the target of obesogens. J Steroid Biochem Mol Biol. 2011;127(1-2):4-8.

79. Newbold RR, Padilla-Banks E, Jefferson WN. Environmental estrogens and obesity. Mol Cell Endocrinol. 2009;304(1-2):84-9.
80. Meeker JD. Exposure to environmental endocrine disruptors and child development. Arch Pediatr Adolesc Med. 2012;166(10):952-8.

81. Heindel JJ, vom Saal FS. Role of nutrition and environmental endocrine disrupting chemicals during the perinatal period on the aetiology of obesity. Mol Cell Endocrinol. 2009;304(1-2):90-6.

82. LaMerrill M, Birnbaum LS. Childhood obesity and environmental chemicals. Mt Sinai J Med. 2011;78(1):22-48.

83. Tang-Peronard JL, Andersen HR, Jensen TK, et al. Endocrinedisrupting chemicals and obesity development in humans: a review. Obes Rev. 2011;12:622-36.

84. Melzer D, Gates P, Osborne NJ, et al. Urinary bisphenol a concentration and angiography-defined coronary artery stenosis. PLoS One. 2012;7(8):e43378.

85. Pandey S, Shetty A, Hamilton M, et al. Obstetric and perinatal outcomes in singleton pregnancies resulting from IVF/ICSI: a systematic review and meta-analysis. Hum Reprod Update. 2012;18(5): 485-503.

86. Ferraretti AP, Goossens V, Kupka M, et al. Assisted reproductive technology in Europe, 2009: results generated from European registers by ESHRE. Hum Reprod. 2013;28(9):2318-31.

87. Sullivan EA, Zegers-Hochschild F, Mansour R, et al. International Committee for Monitoring Assisted Reproductive Technologies (ICMART) world report: assisted reproductive technology 2004. Hum Reprod. 2013;28(5):1375-90.

88. Sunderam S, Kissin DM, Flowers L, et al. Assisted reproductive technology surveillance-United States, 2009. MMWR Surveill Summ. 2012;61(7):1-23.

89. Yeung EH, Druschel C. Cardiometabolic health of children conceived by assisted reproductive technologies. Fertil Steril. 2013;99(2):318-26.

90. Valenzuela-Alcaraz B, Crispi F, Bijnens B, et al. Assisted reproductive technologies are associated with cardiovascular remodeling in utero that persists postnatally. Circulation. 2013;128(13):1442-50.

91. Scherrer U, Rimoldi SF, Rexhaj E, et al. Systemic and pulmonary vascular dysfunction in children conceived by assisted reproductive technologies. Circulation. 2012;125(15):1890-6.

92. Celermajer DS. Manipulating nature: might there be a cardiovascular price to pay for the miracle of assisted conception? Circulation. 2012;125(15):1832-4.

93. Katari S, Turan N, Bibikova M, et al. DNA methylation and gene expression differences in children conceived in vitro or in vivo. Hum Mol Genet. 2009;18(20):3769-78. 